\title{
A cool disk in the Galactic Center?
}

\author{
B. F. Liu, F. Meyer, and E. Meyer-Hofmeister
}

\author{
Max-Planck-Institut für Astrophysik, Karl- Schwarzschildstr. 1, 85740 Garching, Germany
}

Received 30 December 2003 / Accepted 25 March 2004

\begin{abstract}
We study the possibility of a cool disk existing in the Galactic Center in the framework of the disk-corona evaporation/condensation model. Assuming an inactive disk near the gravitational capture distance left over from an earlier evolutionary stage, a hot corona should form above the disk since there is a continuous supply of hot gas from stellar winds of the close-by massive stars. We study the interaction between the disk and the corona. Whether the cool disk can survive depends on the mass exchange between disk and corona which is determined by the energy and pressure balance. If evaporation is the dominant process and the rate is larger than the Bondi accretion rate in the Galactic Center, the disk will be depleted within a certain time and no persistent disk will exist. On the other hand, if the interaction results in hot gas steadily condensing into the disk, an inactive cool disk with little gas accreting towards the central black hole might survive in the Galactic Center. For this case we further investigate the Bremsstrahlung radiation from the hot corona and compare it with the observed X-ray luminosity. Our model shows that, for standard viscosity in the corona $(\alpha=0.3)$, the mass evaporation rate is much higher than the Bondi accretion rate and the coronal density is much larger than that inferred from Chandra observations. An inactive disk can not survive such strong evaporation. For small viscosity $(\alpha \lesssim 0.07)$ we find condensation solutions. But detailed coronal structure computations show that in this case there is too much X-ray radiation from the corona to be in agreement with the observations. From this modeling we conclude that there should be no thin/inactive disk presently in the Galactic Center. However we do not exclude that the alternative non-radiative model of Nayakshin (2004) might instead be realized in nature and shortly discuss this question.
\end{abstract}

Key words. accretion, accretion disks - black hole physics - Galaxy: center

\section{Introduction}

The case for a massive black hole in our Galactic Center coincident with the radio source $\mathrm{Sgr} \mathrm{A}^{*}$ represents a unique opportunity to probe the dynamics of gas accreting onto a massive black hole. Dynamical measurements of stellar velocities within the central 0.1 parsec of the Galactic Center from 10 years of high resolution imaging indicate a central mass of $2.6 \times 10^{6} M_{\odot}$ (Genzel et al. 2000; Ghez et al. 2000) or $3.3 \times 10^{6} M_{\odot}$ (Schödel et al. 2003), recent measurements of stellar orbits (Genzel et al. 2003) provide evidence for a $3.6 \times 10^{6} M_{\odot}$ black hole. The Eddington luminosity for $3.3 \times 10^{6} M_{\odot}$ is $L_{\mathrm{Edd}} \approx 10^{44.6} \mathrm{erg} \mathrm{s}^{-1}$. Observations show that Sgr $\mathrm{A}^{*}$ is an extremely dim galactic nucleus. The luminosity in the submillimeter/far infrared region is $L_{\text {submm }} \sim 10^{-8.6} L_{\text {Edd }}$, even less in the infrared band with $L_{\mathrm{IR}}<10^{-9.6} L_{\mathrm{Edd}}$, also very low is the quiescent $\mathrm{X}$-ray luminosity, $L_{\mathrm{X}}<10^{-11} L_{\mathrm{Edd}}$ (Narayan 2002, where $L_{\text {Edd }}=10^{44.6} \mathrm{erg} \mathrm{s}^{-1}$ ). Chandra observations directly image the hot X-ray-emitting thermal gas in the vicinity of the Bondi accretion radius where the surrounding gas is captured by the gravitational pull of the central black hole, and determine temperatures and densities that allow to estimate a mass accretion rate of $\mathrm{Sgr} \mathrm{A}^{*}$ of $\dot{M}_{\text {Bondi }} \sim(0.3-1) \times 10^{-5} M_{\odot} /$ yr, equivalent to $\sim 10^{-4} \dot{M}_{\text {Edd }}$

Send offprint requests to: B. F. Liu, e-mail: bifang@mpa-garching.mpg.de (with $\dot{M}_{\text {Edd }} \equiv L_{\text {Edd }} / 0.1 c^{2}$ ) (e.g., Baganoff et al. 2003). If mass flows steadily through a Shakura-Sunyaev thin disk (Shakura \& Sunyaev 1973) to the central black hole at this accretion rate, the thin disk model would predict a luminosity $L_{\text {disk }} \sim$ $0.1 \dot{M}_{\text {Bondi }} c^{2} \sim 10^{40.8} \mathrm{erg} \mathrm{s}^{-1} \sim 10^{-4} L_{\text {Edd }}$, much higher than the luminosity in any band observed. Thus, a standard thin disk model appears to be ruled out.

The low luminosity was explained by an advectiondominated accretion flow (ADAF), with a spectral fit first presented by Narayan et al. (1995). In the following years important observational results on the emission of Sgr A* and theoretical work lead to an improved model for radiatively inefficient accretion flows (RIAFs). RIAF models for Sgr A* are discussed in the recent reviews of Yuan et al. (2003) and Quataert (2003). According to these models, most of the thermal energy released by viscosity and increased by compression is retained in the gas and advected to the central black hole. The RIAF model in addition assumes that very little mass from large radii actually accretes onto the black hole while a large part is lost through outflows during the accretion. These models naturally yield the observed spectra of Sgr A*. A key constraint on these models is that the fraction of gravitational energy heating the electrons must be very small and hence a two-temperature treatment of the plasma is required.

Another possibility to explain the low luminosity of Sgr A* might come from the existence of cold molecular gas in the 
parsec region of the Galactic Center in the form of an inactive/dead thin disk without accretion. Falcke \& Melia (1997) had suggested such a "fossil" disk (for a review see Melia \& Falcke 2001). The model assumes that gas captured at Bondi accretion rate condenses onto such an inactive disk without mass accretion onto the black hole. This runs into difficulties because the inflowing gas produces a fair amount of luminosity in the infrared (Narayan 2002) as it clashes onto the disk and loses its thermal and kinetic energy. This radiation is not seen. Nayakshin (2004) revisits the concept of an inactive disk. He suggests that, in the case of an extraordinarily long mean free path (larger than the pressure scale of the corona) and extremely low viscosity of the hot gas, the energy can be conducted to a very thin transition layer by free streaming electrons and is then radiated in infrared to UV wavelengths. As this radiation from the thin layer would be observed edge-on a discrepancy between predicted and observed luminosities could be avoided. This interesting suggestion deserves further analysis of the transition between the hot gas and the cool layer and the coupling between the hot ions and the energy transferring electrons.

As a further contribution to the issue of a cool disk around the Galactic Center we here study the vertical structure of a hot corona in interaction with a disk below. We assume a cool disk in the outer region around the circularization radius where the free fall of a Bondi type accreting hot gas goes over into a circular motion around the gravitational center due to its specific angular momentum. Such a disk might not be unreasonable since the system must have been quite bright during an earlier evolutionary stage and angular momentum that was released by the high accretion rate should have moved disk mass into outward regions. With winds from young stars being captured by the gravitational field of the black hole, a corona unavoidably forms above the cool disk. The question is, how do disk and corona evolve? Does the hot gas condense to the disk with little mass actually accreting to the black hole, or does mass rather evaporate from the disk to the corona overwhelming the incoming hot gas and even finally depleting the cool disk underneath?

The answer depends mainly on the rate at which the hot gas is supplied from the capture radius. If gas is supplied to the corona at a sufficiently high rate coronal gas condenses to the cool disk. If no gas is supplied from the outside or if the outside gas supply is too small mass instead evaporates from the disk into the corona. Both processes are the consequence of pressure and energy equilibrium between the disk and the corona (Meyer et al. 2000; Liu et al. 2002). Here we study in detail the structure of the corona for the case of the Galactic Center in order to see what the dominant process between disk and corona is, condensation or evaporation. Can the cool disk survive if evaporation is dominant? Furthermore, the detailed computation allows to calculate the Bremsstrahlung luminosity of the corona and compare it with the observed X-ray luminosity. We show that these results exclude the existence of any cool disk in our Galactic Center.

In Sect. 2 we describe the physics of the interaction between the disk and the corona. In particular we discuss how a radial inflow of mass from the outside affects the mass and energy balance in such a corona. In Sect. 3 we present numerical results and show how the value of the viscosity affects evaporation or condensation. We discuss several aspects of our results in Sect. 4, including a comparison with a non-radiative condensation model of Nayakshin (2004) and the question of a past disk being evaporated now in the Galactic Center. A conclusion follows in Sect. 5.

\section{The physics of interaction between disk and corona}

For a hot corona lying above a cool disk, interaction between the disk and the corona occurs via energy and mass exchange. The hot corona conducts heat downward by electrons. At the bottom the heat is radiated away. If the density in the corona is too low, Bremsstrahlung is inefficient and the thermal conductive flux heats up some of the disk gas leading to mass evaporation from the disk into the corona. The resulting density increase in the corona raises the radiation loss and thereby counteracts further evaporation. If the coronal density is too high, radiative cooling is too strong and gas condenses into the disk. At the final equilibrium density, cold gas steadily evaporates from the disk into the corona if mass is drained continuously from the corona inward by diffuse flow, or hot gas steadily condenses to the disk if the corona continuously gains mass by mass flow. For example, when there is no hot gas coming in through the outer boundary, (case 1), mass is continuously lost from the corona by accretion towards the central object. This is resupplied by evaporation from the surface of the cool disk as the corona tries to restore the density to the equilibrium level. If there is more hot gas being fed in at the outer boundary than what flows inward towards the center, (case 2), hot gas continuously condenses to the cool disk.

\subsection{Basic equations}

The equations describing the corona above the disk are derived in our earlier studies (Meyer \& Meyer-Hofmeister 1994; Meyer et al. 2000; Liu et al. 2002). For the Galactic Center, we consider the region around the circularization radius $10^{4}-10^{5} R_{\mathrm{S}}$ ( $R_{\mathrm{S}}$ Schwarzschild radius) far from the black hole. In this region, electrons are collisionally well coupled to ions, temperatures of electrons and ions are the same.

We now discuss the upper boundary condition for the corona at such radii. The earlier investigations showed that in general wind loss from the corona is an integral part of the solution, where a sonic transition occurs at some height and the wind flow cross section flares out with the wind expansion. In an advanced multi-zone modeling (Meyer-Hofmeister \& Meyer 2003) we found that the wind pressure is highest at the distance where the evaporation efficiency is highest, at a few hundred Schwarzschild radii. The pressure in the expanding wind from this region even dominates over the pressure at a sonic point of winds from farther out regions and prevents sonic transition and wind loss there altogether. Thus for evaporation solutions in these outer regions we apply the condition of zero vertical velocity at a height of $z=R$. The actual height 
at this point is not important as long as it includes the lower down region where most of the coronal action occurs.

In the case of condensation solutions the incoming mass flow from the outside accretion anyhow dominates the coronal pressure and prevents any free wind expansion. We thus can apply the same boundary condition. In our equations we can also leave out the so-called flaring terms which are only important in the wind expansion geometry.

In the following we list the four ordinary differential equations describing the coronal flows above a disk in the Galactic Center.

Continuity of mass flow

$$
\frac{\mathrm{d}}{\mathrm{d} z}\left(\rho v_{z}\right)=\frac{2}{R} \eta_{\mathrm{M}} \rho v_{R}
$$

$z$-component of the equation of motion

$\rho v_{z} \frac{\mathrm{d} v_{z}}{\mathrm{~d} z}=-\frac{\mathrm{d} P}{\mathrm{~d} z}-\rho \frac{G M z}{\left(R^{2}+z^{2}\right)^{3 / 2}}$.

Energy equation

$$
\begin{aligned}
\frac{\mathrm{d}}{\mathrm{d} z} & {\left[\rho v_{z}\left(\frac{v^{2}}{2}+\frac{\gamma}{\gamma-1} \frac{P}{\rho}-\frac{G M}{\left(R^{2}+z^{2}\right)^{1 / 2}}\right)+F_{\mathrm{c}}\right] } \\
= & \frac{3}{2} \alpha P \Omega-n_{\mathrm{e}} n_{\mathrm{i}} L(T) \\
& +\frac{2}{R} \eta_{\mathrm{E}} \rho v_{R}\left(\frac{v^{2}}{2}+\frac{\gamma}{\gamma-1} \frac{P}{\rho}-\frac{G M}{\left(R^{2}+z^{2}\right)^{1 / 2}}\right) .
\end{aligned}
$$

Thermal conduction for a fully ionized plasma

$$
F_{\mathrm{c}}=-\kappa_{0} T^{5 / 2} \frac{\mathrm{d} T}{\mathrm{~d} z}
$$

with $\kappa_{0}=10^{-6} \mathrm{erg} \mathrm{s}^{-1} \mathrm{~cm}^{-1} \mathrm{~K}^{-7 / 2}$ (Spitzer 1962).

Here $\rho, P, T$ are density, pressure and temperature, $v_{R}$ and $v_{z}$ the radial and vertical velocity, $M$ is the black hole mass, $G$ the gravitational constant and $\Omega$ the rotational frequency, $n_{\mathrm{e}}$ and $n_{\mathrm{i}}$ are electron and ion particle densities, $n_{\mathrm{e}} n_{\mathrm{i}} L(T)$ is the Bremsstrahlung cooling rate, and $\gamma$ the ratio of specific heats. $\alpha$ is the viscosity parameter (ratio of viscous stress to pressure). The terms $\eta_{\mathrm{M}}$ and $\eta_{\mathrm{E}}$ account for radial mass and energy flows with $\eta_{\mathrm{E}}=\eta_{\mathrm{M}}+0.5$, explained in the next section. The difference between $\eta_{\mathrm{M}}$ and $\eta_{\mathrm{E}}$ results from the fact that the specific energy which the mass flow carries scales radially as $\frac{1}{r}$. We consider stationary azimuthally symmetric flows.

Compton cooling is negligible for the coronal structure at distances of $10^{4}$ to $10^{5}$ Schwarzschild radii. Three possible contributions to Compton cooling have to be considered. (1) The cooling by the radiation from the disk surface caused by mass flow in the disk was investigated by Liu et al. (2002) and shown to be negligible even for mass flow rates in the disk of $0.02 \dot{M}_{\text {Edd. }}$. (2) We have estimated that the energy loss by Compton cooling from radiation caused by reprocessing of coronal X-rays is always less than $0.3 \%$ of that by bremsstrahlung. (3) The Compton effect from the X-rays from the central source and the surrounding gas is negligible in our context because of the very low observed radiation.
At the lower boundary $z_{0}$ we start our calculations at the temperature $T=10^{6.5} \mathrm{~K}$. This value is in the steep temperature profile in a thin transition zone of nearly constant pressure. Its physics can be described by the balance between gain of heat by thermal conduction and radiation loss. Other effects like frictional heating and energy transport by the vertical mass flow are negligible here (Meyer et al. 2000). This establishes a relation between temperature and heat flux which can be scaled according to the pressure (Smeleva \& Syrovatskii 1973). If one temperature in the profile is selected then, due to the scaling, one obtains a unique relation between thermal heat flux and pressure (Liu et al. 1995). We use this relation

$F_{\mathrm{c}}=-2.73 \times 10^{6} \mathrm{P}$ in cgs units.

As discussed above at the upper boundary $z=R$ we take

$F_{\mathrm{c}}=0$ and $v_{z}=0$.

\subsection{Parameter $\eta_{\mathrm{M}}$ for the net inflow of mass}

The parameters $\eta_{\mathrm{M}}$ and $\eta_{\mathrm{E}}$ are introduced to account for the radial mass and energy flows (Meyer-Hofmeister \& Meyer 2003). In the early evaporation model ("one-zone-model", Meyer \& Meyer-Hofmeister 1994), it is assumed that essentially all the mass evaporating from the disk to the corona flows towards the farther inward coronal region and that there is no significant mass flow into the corona from outside. In our designation this is $\eta_{\mathrm{M}}=1$. Taking into account the structure of the radially neighboring coronal areas and the mass and energy exchange with these we have to consider the actual radial gradients of mass flow in the vertical structure equations. The introduction of $\eta_{\mathrm{M}}$ is to parameterize the unknown radial gradient in the general mass conservation equation, $\frac{\partial}{\partial z}\left(\rho v_{z}\right)+\frac{1}{R} \frac{\partial}{\partial R}\left(R \rho v_{R}\right)=0$. We approximate this in Eq. (1) with a height-averaged factor $\eta_{\mathrm{M}}$, which implies,

$\eta_{\mathrm{M}} \approx \frac{\dot{M}\left(R_{\text {in }}\right)-\dot{M}\left(R_{\text {out }}\right)}{\dot{M}(R)}$,

where $\dot{M}\left(R_{\text {out }}\right)$ is the incoming mass flow rate from the outer boundary of the one-zone corona, $\dot{M}\left(R_{\text {in }}\right)$ mass outflow rate at the inner boundary, and $\dot{M}(R)$ the typical mass flow rate in the one-zone corona. Thus, the parameter $\eta_{\mathrm{M}}$, by its definition, depends on the net mass gain/loss rate through the radial boundaries.

Integration of Eq. (1) along the $z$-direction gives,

$\eta_{\mathrm{M}} \dot{M}(R)=2 \pi R^{2} \dot{m}_{0}$,

where $\dot{m}_{0}$ is mass evaporation rate $\left(\dot{m}_{0}>0\right)$ or condensation rate $\left(\dot{m}_{0}<0\right)$ per unit area at the interface between disk and corona. Obviously, $\eta_{\mathrm{M}}>0$ describes the fraction of the coronal mass flow contributed by disk evaporation, $\eta_{\mathrm{M}}<0$ describes the fraction of the coronal flow condensing to the disk. A special case $\eta_{\mathrm{M}}=0$ means there is no mass evaporation or condensation between disk and corona. If there is any gas coming from the outer boundary, all of this flows through the corona towards the central black hole. Two extreme cases are $\eta_{\mathrm{M}}=1$ and $\eta_{\mathrm{M}}=-1$. The former represents the case when no mass enters through the outer boundary and all the mass flowing in the 
corona is contributed by the evaporation, $\dot{M}(R)=2 \pi R^{2} \dot{m}_{0}=$ $\dot{M}\left(R_{\text {in }}\right)$. The latter case represents the situation in which no mass flows towards the central object at the inner boundary and all the mass entering the corona from the outer boundary condenses to the disk, $\dot{M}(R)=\dot{M}\left(R_{\text {out }}\right)=-2 \pi R^{2} \dot{m}_{0}$. Therefore, our parameter $\eta_{\mathrm{M}}$ lies in the range $-1 \leq \eta_{\mathrm{M}} \leq 1$.

\section{Condensation or evaporation of gas in the central arcsecond of our Galactic Center?}

In our Galactic Center, hot gas in the central parsec produced by winds from massive stars is gravitationally captured by the black hole at a distance around 0.04 parsec which corresponds to 1 arcsec at the sky. If there is a cool (inactive) disk in this region, a corona forms above the disk, fed by the captured hot gas. The situation is a bit different from the one discussed in our previous evaporation model, where we assumed that cold gas flows inward from the outer region (e.g. a secondary star) via the thin disk and then feeds the corona through evaporation. There it was assumed that essentially no significant hot gas comes from a further outward located hot corona.

Now the situation is turned around, hot gas accretes through the corona without any cold gas being fed into the cool disk from the outer disk boundary. As explained in the last section, if much mass is fed into the corona, the pressure in the corona is high. The pressure and energy balance between disk and corona leads to mass condensing from the corona to the disk. On the other hand, if the feeding rate to the corona is low, mass evaporation from the disk to the corona is the dominant process. Strictly speaking, how large the mass exchange rate is and whether mass evaporates or condenses depend on the rate of net mass inflow into the coronal region, i.e., the difference between the mass feeding rate at the outer boundary and the mass loss rate to the central object at the inner boundary.

If the equilibrium between disk and corona leads to strong mass evaporation, the cool disk will eventually be depleted and mass then accretes via a hot corona/ADAF or RIAF. If condensation dominates, the hot gas captured by the gravitational field of the black hole finally flows to the disk and is deposited there with little mass actually accreting onto the black hole. In the following we investigate these coronal features for the case of our Galactic Center.

\subsection{Standard viscosity $\alpha=0.3$}

At the Galactic Center, hot gas accretes at a Bondi accretion rate of $\sim 10^{-4} \dot{M}_{\text {Edd }}$ from the capture radius $R \sim 10^{5} R_{\mathrm{S}}$ (0.8 arcsec), with the circularization radius estimated as $R \sim$ $10^{4} R_{\mathrm{S}}$. The circularization radius might also be larger, e.g. if the accreted winds predominantly come from close-by stars orbiting in a common sense around the Galactic Center. We thus investigate the coronal features in a region from $10^{4} R_{\mathrm{S}}$ to $10^{5} R_{\mathrm{S}}$ according to the scenario described in the section above. For our computations we took the mass of the black hole as $3.3 \times 10^{6} M_{\odot}$. We first discuss the results for $\alpha=0.3$. For this value our earlier modeling of hard/soft spectral transitions in $\mathrm{X}$-ray binaries and applications to AGN gave good quantitative comparisons with observations. Henceforth we call it the

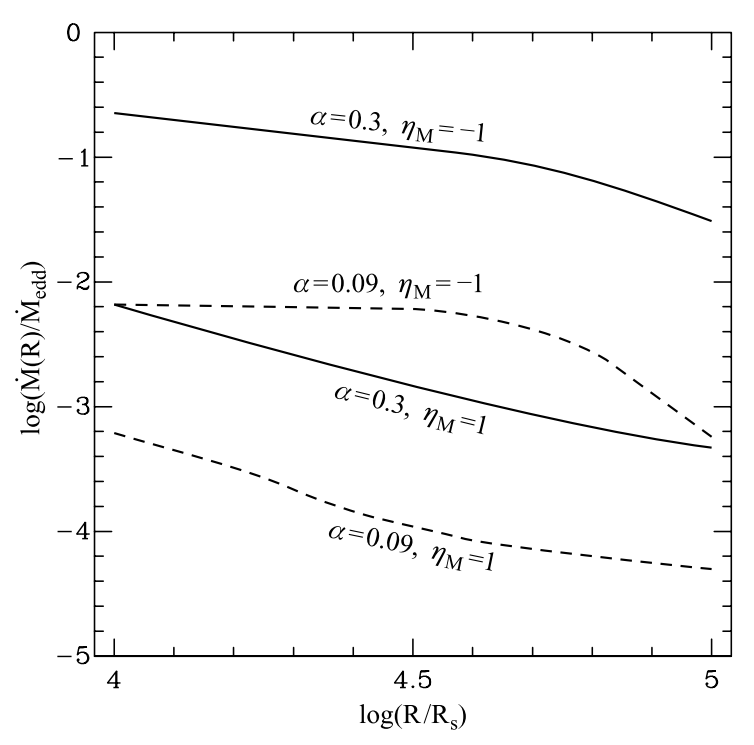

Fig. 1. Radial mass flow rate in the corona for given parameters $\eta_{\mathrm{M}}=1$, evaporation, and $\eta_{\mathrm{M}}=-1$, condensation; Solid lines: solutions for standard viscosity, $\alpha=0.3$, dashed lines solutions for a lower viscosity $\alpha=0.09$. Note that the curves do not represent a distribution of the radial mass flow in the corona, but give the typical mass flow rate in the local corona at the distance $R / R_{\mathrm{S}}$ under the conditions specified with $\eta_{\mathrm{M}}$.

"standard value". A similar value was also chosen for the application of ADAFs to luminous black hole X-ray binaries (Esin et al. 1997).

\subsubsection{Evaporation model}

In our terminology any value of $\eta_{\mathrm{M}}>0$ means there is more gas flowing out of the coronal region than coming in. The net mass flow out of the corona is contributed by mass evaporation from the disk. The case of "standard evaporation", considered in the one-zone-model corresponds to $\eta_{\mathrm{M}}=1$, which means that the typical mass flow in the corona is the same as the mass evaporation into the corona at that distance.

Figure 1 shows the radial mass flow rate in the corona (on both sides of the disk) for distances $10^{4} R_{\mathrm{S}}$ to $10^{5} R_{\mathrm{S}}$. The mass flow rate is $\dot{M}(R)=-2 \int_{z_{0}}^{z_{1}} 2 \pi R \rho v_{R} \mathrm{~d} z$, with $v_{R} \approx-\alpha V_{\mathrm{s}}^{2} / \Omega R$ the typical radial drift velocity $\left(V_{\mathrm{s}}\right.$ isothermal sound speed, $\Omega$ Kepler angular frequency, $z_{0}$ and $z_{1}$ lower and upper boundary of the corona respectively).

The lower solid line represents the mass accretion rate for the standard one-zone evaporation model. We see that the mass flow rates in the coronal region from $10^{4} R_{\mathrm{S}}$ to $10^{5} R_{\mathrm{S}}$ are around $10^{-3} \dot{M}_{\text {Edd }}$, about 10 times higher than the incoming mass flow estimated from Bondi accretion and Chandra observations as $\dot{M} \sim 10^{-4} \dot{M}_{\text {Edd }}$. This indicates that the dominant process in the disk-corona system is that gas evaporates from the disk to the corona and then flows towards the central black hole. The hot gas captured at the Bondi radius is only a minor contribution and hardly affects the coronal structure. A strong corona with high density and high temperature is built up above the disk by the mass evaporation. 

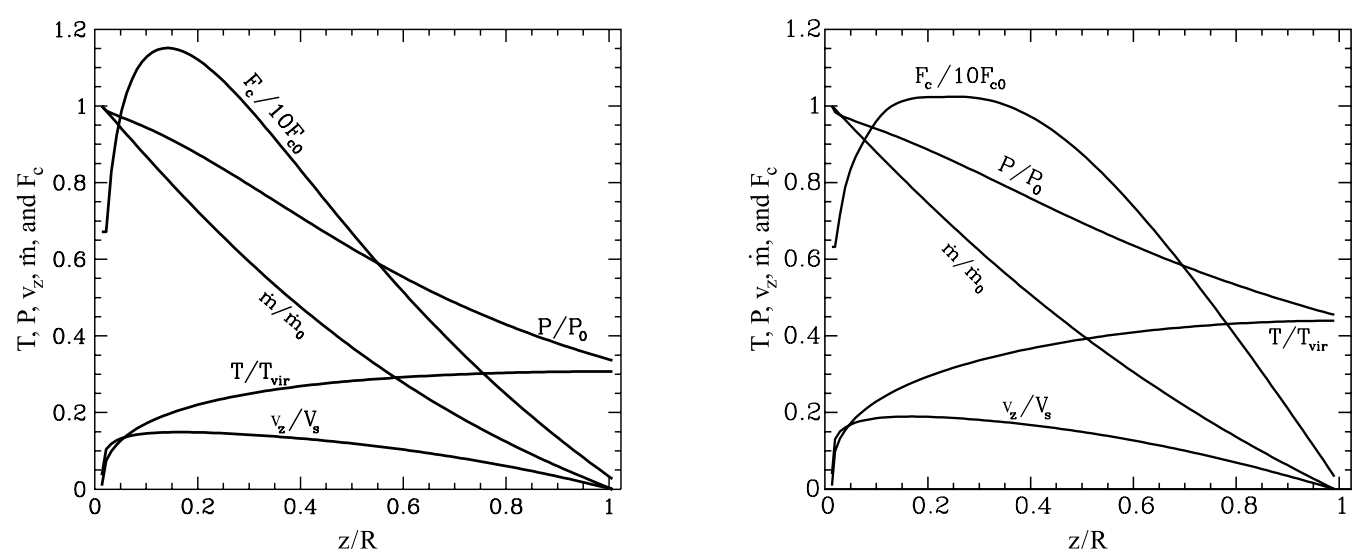

Fig. 2. Vertical structure of the corona with mass evaporation from the disk to the corona $\left(\eta_{\mathrm{M}}=1\right.$, left panel $)$ and with mass condensation from the corona to the disk $\left(\eta_{\mathrm{M}}=-1\right.$, right panel) at the distance $R=10^{4} R_{\mathrm{S}}$ from a central black hole of $3.3 \times 10^{6} M_{\odot} . z / R$ ratio of height above midplane to distance. Distribution of the following quantities: temperature $T$ in units of virial temperature $T_{\text {vir }}$, pressure $P$, thermal conductive flux $F_{\mathrm{c}}$ and vertical mass flow rate $\dot{m}=\rho v_{z}$ (the latter ones scaled to their values at the lower boundary: $P_{0} \approx 1.9 \times 10^{-2}$ dyn $\mathrm{cm}^{-2}$, $F_{\mathrm{c} 0} \approx-5.2 \times 10^{4} \mathrm{erg} \mathrm{cm}^{-2} \mathrm{~s}^{-1}, \dot{m}_{0} \approx 3.4 \times 10^{-11} \mathrm{~g} \mathrm{~cm}^{-2} \mathrm{~s}^{-1}$ for the left panel, and $P_{0} \approx 0.6 \mathrm{dyncm} \mathrm{cm}^{-2}, F_{\mathrm{c} 0} \approx-1.6 \times 10^{5} \mathrm{erg} \mathrm{cm}^{-2} \mathrm{~s}^{-1}$, $\dot{m}_{0} \approx-10^{-9} \mathrm{~g} \mathrm{~cm}^{-2} \mathrm{~s}^{-1}$ for the right panel).

Figure 2 shows the coronal structure at $R=10^{4} R_{\mathrm{S}}$ for both cases, evaporation and condensation. From the figure we see that in the case of evaporation the typical temperature of the coronal gas is $\sim 0.3 T_{\text {vir }} \sim 10^{8} \mathrm{~K}$ (virial temperature $T_{\text {vir }}=G M /\left(R \frac{\mathfrak{R}}{\mu}\right), \mathfrak{R}$ gas constant, $\mu$ molecular weight, taken as 0.62 ). The particle number density which follows from pressure and temperature is larger than $5 \times 10^{5} \mathrm{~cm}^{-3}$. Temperature and density both are much larger than the values observed by Chandra at the Galactic Center. A disk might have existed earlier but it could have been eventually depleted by the mass evaporation since there is no mass supply for the cool disk. Therefore, the standard disk-corona evaporation model appears to exclude the continued existence of a cool disk in the Galactic Center. Note that this argument is independent on whether the cool disk is completely inert or whether it allows some mass flow itself.

\subsubsection{Condensation model}

To let coronal gas condense to the disk, we need high pressure in the corona. This requires more gas feeding into the corona from outside than gas flowing out of the corona at the inner boundary. The value of $\eta_{M}$ is then negative. An extreme case we are interested in for our investigation is that all the gas coming in from the outer boundary condenses to the disk without any coronal accretion toward the central object, $\eta_{\mathrm{M}}=-1$. The mass flow rates in the coronal region at distances $10^{4} R_{\mathrm{S}}$ to $10^{5} R_{\mathrm{S}}$ are shown in Fig. 1 as upper solid line. Obviously, the derived mass flow rate $\dot{M}\left(R_{\text {out }}\right)=-\eta_{\mathrm{M}} \dot{M}(R)=\dot{M}(R)$ with condensation $\left(\eta_{\mathrm{M}}=-1\right.$ prescribed $)$, is higher than that with mass evaporation $\left(\eta_{\mathrm{M}}=1\right.$ prescribed).

The condensation rate is far too high compared to the Bondi accretion rate in the Galactic Center. The density in such a corona is also much higher than the value inferred from the observations. Therefore, we cannot expect that, if there is a cool disk in the Galactic Center, hot gas captured by the black hole at the Bondi radius mainly condenses into the disk and is deposited there. Instead, the disk gas will evaporate into the

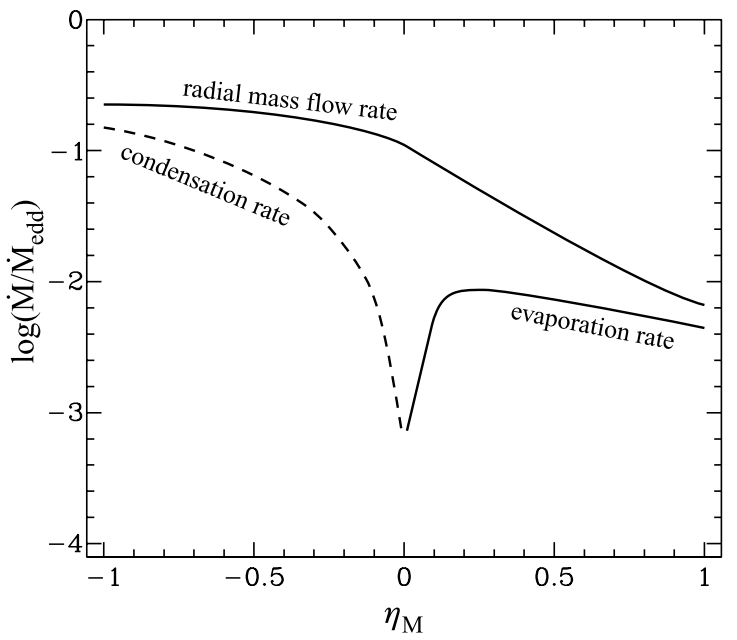

Fig. 3. Radial mass flow rate in the corona (upper curve) together with condensation rate $=-2 \pi R^{2} \dot{m}_{0} / \dot{M}_{\text {Edd }}$ (dashed line), and evaporation rate $=2 \pi R^{2} \dot{m}_{0} / \dot{M}_{\text {Edd }}$ (lower solid line), for the Galactic Center at $R=$ $10^{4} R_{\mathrm{S}}$ (viscosity parameter $\alpha=0.3$ ). At $\eta_{\mathrm{M}}=-1$ all mass that flows in the corona has come from the outside and condenses into the cool disk, then with increasing $\eta_{\mathrm{M}}$ a smaller part settles into the disk until at $\eta_{\mathrm{M}}=0$ no mass condenses. At $\eta_{\mathrm{M}}=1$ all mass flowing inward in the corona has evaporated from the cool disk (for details see text).

corona, increasing the coronal flow inward significantly. As noted above, this process can finally deplete the cool disk. From then on no cool disk exists anymore in the Galactic Center.

\subsubsection{Dependence of the solutions on $\eta_{\mathrm{M}}$}

More generally, we study the condensation and evaporation solutions for a sequence of values of $\eta_{\mathrm{M}}$. Figure 3 shows how the mass flow rate and the evaporation/condensation rate at $R=10^{4} R_{\mathrm{S}}$ change with the value of the parameter $\eta_{\mathrm{M}}$. The results show that condensation solutions are connected with a high mass flow rate through the coronal region. At $\eta_{\mathrm{M}}=-1$ all mass that flows in the corona has come from the outside and condenses into the cool disk. With increasing $\eta_{\mathrm{M}}$ a 
smaller and smaller part of the coronal mass flow settles into the disk until at $\eta_{\mathrm{M}}=0$ no mass condenses and all mass that comes from the outside continues inward. For increasing positive $\eta_{\mathrm{M}}$ mass evaporation contributes a growing fraction to the mass flow in the corona until at $\eta_{\mathrm{M}}=1$ all mass flowing inward in the corona comes from evaporation of the cool disk. (The difference between upper and lower curve at $\eta_{\mathrm{M}}=-1$ and 1 is an artifact of the one-zone model approximation and results from the different way in which averages over the onezone area are defined.) One may note that as the characteristic mass flow rate is proportional to the pressure in the corona, $\dot{M}(R)=-2 \int_{z_{0}}^{z_{1}} 2 \pi R \rho v_{R} \mathrm{~d} z \propto \int_{z_{0}}^{z_{1}} P \mathrm{~d} z$, the results in Fig. 3 reflect the intrinsic relation: more pressure means more radiative cooling which supports condensation, less pressure means less radiative cooling and supports evaporation.

Thus, if we know the pressure (or mass content) in a coronal region, our computations for the equilibrium between disk and corona allow to derive how much mass condenses or evaporates. This is not directly determined by the incoming mass flow alone, but depends on the net mass flow into the region, i.e., the difference of incoming mass flow at the outer boundary and the outgoing mass flow at the inner boundary. Therefore, when we know both the inner and outer boundary conditions as in multi-zone modeling (Meyer-Hofmeister \& Meyer 2003), we are able to determine the vertical and the radial structure of the corona. Otherwise, $\eta_{\mathrm{M}}$ will be an open parameter in the range of -1 and 1 .

Combining Figs. 1 and 3, we find that, for $-1 \leq \eta_{\mathrm{M}} \leq 1$, the mass flow rate in the corona is between the two solid curves of Fig. 1. The same is true for all distances of $10^{4} R_{\mathrm{S}} \lesssim R \lesssim$ $10^{5} R_{\mathrm{S}}$. In other words, no matter how large a fraction of the coronal mass flow condenses to the disk $\left(\eta_{\mathrm{M}}\right.$ between -1 and 0 ), or how large a fraction of the coronal mass flow originates from disk evaporation $\left(\eta_{\mathrm{M}}\right.$ between 0 and 1), the mass flow in the corona would be much larger than the Bondi accretion rate of $10^{-4} M_{\text {Edd }}$ inferred from observations. The detailed calculations also show that the hot gas density is much larger than that obtained from the observations.

As a consequence of such a large mass flow rate, the luminosity of coronal radiation in X-rays (note that it is not in the infrared), estimated from $L \sim G M \dot{M}(R) / 2 R=$ $2.5\left(R / R_{\mathrm{S}}\right)^{-1}\left[\dot{M}(R) / \dot{M}_{\mathrm{Edd}}\right] L_{\mathrm{Edd}}$, is very high. For instance, at $R \sim 10^{5} R_{\mathrm{S}}, \dot{M}(R) \sim 10^{-3} \dot{M}_{\mathrm{Edd}}$, the radiation from the corona would then be $L \sim 2.5 \times 10^{-8} L_{\text {Edd }}$, much larger than the observed quiescent X-ray luminosity $L_{X} \sim 10^{-11} L_{\text {Edd }}$. The Bremsstrahlung radiation is confirmed by detailed computations of the coronal structure.

Therefore, for standard $\alpha$, these consistent disk-corona model calculations exclude a disk at distances $10^{4} R_{\mathrm{S}} \lesssim R \lesssim$ $10^{5} R_{\mathrm{S}}$ from the Galactic Center by comparison of mass flow rate, density, and luminosity of the hot gas predicted by theory with those actually observed.

\subsection{Low viscosity}

In the last section we showed that for standard $\alpha$ no condensation solution exists that is compatible with the observed mass accretion rate in the Galactic Center. We investigate whether agreement between observation and a disk-corona analysis can be achieved for smaller values of $\alpha$.

In the models the viscosity parameter enters directly into the radial drift velocity and the viscous release of heat, both of which become smaller with smaller $\alpha$. Since radiative cooling is not affected by this change in $\alpha$ the balance between heating and cooling requires less pressure in the corona. As a consequence one expects that pressure, temperature, and radial mass flow in the corona decrease when $\alpha$ becomes smaller (Meyer-Hofmeister \& Meyer 2001).

This effect is shown in Fig. 1 where the dashed lines give the mass flow rates in the corona for a smaller viscosity $\alpha=0.09$. The upper and lower dashed lines show the condensation $\left(\eta_{\mathrm{M}}=-1\right)$ and the evaporation solutions $\left(\eta_{\mathrm{M}}=1\right)$ for $10^{4} \leq R / R_{\mathrm{S}} \leq 10^{5}$. For other values of $\eta_{\mathrm{M}},-1<\eta_{\mathrm{M}}<1$, the mass flow rates lie between these two lines. Indeed, a small viscosity in the corona results in a significantly decreased mass flow rate. Density and pressure in the corona also are smaller. For very small $\alpha$, the mass flow rate in the corona can then, in principle, become comparable with the capture rate of $\sim 10^{-4} \dot{M}_{\text {Edd }}$.

\subsubsection{Evaporation model}

In the case of evaporation, $\eta_{\mathrm{M}}>0$, the mass flow rate $\dot{M}\left(R_{\mathrm{in}}\right)$ leaving the coronal region at its inner boundary is always larger than the incoming flow rate $\dot{M}\left(R_{\text {out }}\right)$ at its outer boundary. Thus an energy of the order of $1 / 2$ the gravitational potential energy of the accretion rate is released. Since the temperature of the corona is some tens of a percent of the virial temperature, i.e. $T \sim 10^{8} \mathrm{~K}$ at the distance $R \sim 10^{4} R_{\mathrm{S}}$ and $T \sim 10^{7} \mathrm{~K}$ at the distance $R \sim 10^{5} R_{\mathrm{S}}$, the coronal radiation is in the $\mathrm{X}$-ray range and the corona should have a luminosity $L_{\mathrm{X}} \sim 2.5 \times 10^{-9} L_{\text {Edd }}$ as long as there is hot gas flowing inward at the Bondi accretion rate $\dot{M}=10^{-4} \dot{M}_{\text {Edd }}$ at $R=10^{5} R_{\mathrm{S}}$ and higher at smaller radii. This is 2 orders of magnitude larger than the observed X-ray luminosity. Thus, for any small $\alpha$, the disk evaporation solution can not be consistent with the observations.

\subsubsection{Condensation model}

For condensation solutions part or in the extreme case all of the incoming mass flow $\dot{M}\left(R_{\text {out }}\right)$ would settle through the corona into the cool disk and only a fraction or nothing at all would flow inward through the inner boundary. In any of these cases again an amount of energy of the order of $1 / 2$ gravitational potential energy of the incoming gas is released as heat. One thus has a similar large discrepancy between observed and predicted luminosities.

If $\dot{M}\left(R_{\text {out }}\right)$ is the Bondi accretion rate in the Galactic Center, $\dot{M}\left(R_{\text {out }}\right)=10^{-4} \dot{M}_{\text {Edd }}$, consistency with a condensation solution $\left(-1<\eta_{\mathrm{M}}<0\right)$ can only be obtained by assuming a small viscosity parameter $\alpha$ (for example, $\alpha=0.07$ for $\eta_{\mathrm{M}}=-1$ at $R=10^{5} R_{\mathrm{S}}$ and an even smaller value at smaller distances). However, in either of the extreme cases, $\dot{M}\left(R_{\text {in }}\right) \approx \dot{M}\left(R_{\text {out }}\right)$ or $\dot{M}(R) \approx \dot{M}\left(R_{\text {out }}\right)$, or any case between those, $-1<\eta_{\mathrm{M}}<0$, 
our detailed computations of the coronal structure show that the X-ray luminosity produced by Bremsstrahlung radiation in the corona by far exceeds the luminosity observed. The existence of a cool disk in the Galactic Center seems thus to be excluded. For a condensation picture this was already pointed out by Narayan (2002).

In addition to this result for the situation in the Galactic Center with the presently observed mass accretion rate the results of our investigation allow to discuss whether a formerly existing accretion disk in the context of gravitational instability and star formation in this disk has disappeared due to evaporation until now.

\section{Discussion}

\subsection{Coronal winds}

We have here investigated solutions for which no wind escapes from the corona. This is e.g. the case if winds from farther-in coronal regions fill the "coronal sky" with pressure that prevents the escape of winds from outer regions. We have estimated that this is the case already for winds that originate from the innermost cool disk region at $R=2 \times 10^{3} R_{\mathrm{S}}$ (the distance at which a presumptive disk heated only by its coronal energy release is still cool enough to prevent a magnetic dynamo and magnetic friction).

Should a presumptive cool disk however be cut off already at about $10^{4}$ Schwarzschild radii the coronal solution has to allow for the escape of a wind (if not suppressed by the accreting gas ram pressure). Such a solution has already been obtained earlier (Meyer et al. 2000, for the case $\eta_{\mathrm{M}}=+1$, "the weakest" coronal radiation case). In this case the corona in the upper layers is more tenuous and and the predicted X-ray radiation is somewhat less but with $L_{\mathrm{X}} \approx 10^{37.1} \mathrm{erg} / \mathrm{s}$ still far above the value of $10^{33.6} \mathrm{erg} / \mathrm{s}$ allowed by Chandra observations (Baganoff et al. 2003).

\subsection{Coupling of electrons and ions in the corona}

As shown in Sect. 3 (see also Fig. 1) a small viscosity is required if the density in the corona should not be much higher than inferred from the observations. Our condensation solution shows that, in this case (say, $\alpha=0.09$ ), the temperature in the corona is about $1 / 3$ of the virial temperature and the gas density is fairly low. In such a tenuous hot gas the mean free path can become quite large. Thus the question arises whether the Spitzer formula $\left(F_{\mathrm{c}}=-\kappa_{0} T^{5 / 2} \mathrm{~d} T / \mathrm{d} z\right)$ can still be applied for the thermal conduction and whether the electrons are wellcoupled to the ions so that temperature equilibrium between the two species is established. In our vertical structure computations we therefore compare, at every step of the vertical integration, the conductive heat flux $\kappa_{0} T^{5 / 2} \mathrm{~d} T / \mathrm{d} z$ with the thermal flux of "free streaming" electrons $\frac{3}{2} P V_{\mathrm{s}}$.

We find that for our solutions, the thermal flux given by the Spitzer formula never exceeds the saturated value for free streaming electrons and the mean free path remains always less than the thermal scale height $(T /(\mathrm{d} T / \mathrm{d} z))$. Since $F_{\mathrm{c}}=0$ is required at the upper boundary, as the density becomes smaller and smaller in the upper layers, the temperature gradient approaches zero. This results in a very small heat flux (from the Spitzer formula), which is smaller than the "free-streaming" heat flux. Though the mean free path is quite large at these upper layers it also remains smaller than the temperature scale height which approaches infinity.

We also compared the time needed for equipartition temperature between electrons and ions with the thermal timescale and confirmed that the former is always shorter than the latter. This means that in such a corona, far from the central black hole, electrons and ions are well coupled. Therefore, these models for the corona above a cool disk around the Galactic Center are self-consistent.

\subsection{The effect of magnetic fields}

Could magnetic fields affect our solutions? The standard $\alpha$ description is generally taken as describing the effect of dynamo created magnetic fields, here in our case in the corona. Could additional fields from an underlying disk affect these solutions? Such an effect was discussed by Meyer \& Meyer-Hofmeister (2002) for low luminosity AGN where it was shown that it can have a significant effect on the truncation of accretion disks. The temperature of the disk around Sgr A* from reprocessed coronal radiation, $<10^{2.8} \mathrm{~K}$, however, is too low to allow a dynamo to operate.

\subsection{Alternative model}

Recently, Nayakshin (2004) discussed an alternative model which suggests that all the hot gas captured by the black hole is deposited into an inactive disk (see also Nayakshin 2003). In that model, the corona is extremely tenuous so that the electron mean free path becomes large compared to the pressure scale height of the corona. Thus, a "free-streaming electron" thermal conduction law is applied instead of the classic Spitzer formula. The corona is approximated as a homogeneous column on top of a very thin transition region to the cool disk, in contrast to the vertically layered corona discussed in this paper. The free-streaming particles of long mean free path are thought to transport the thermal energy into the cool disk at the saturation speed of the order of the sound velocity in the corona, thus the coronal gas would be able to sink down into the cool disk at a fraction of that speed and condense as tenuous gas without significant radiation in the X-ray band, low enough not to get into conflict with the observed low X-ray luminosity.

This model lies in a quite different parameter space as the the one discussed in our paper. It differs in the assumption of a very thin transition between the tenuous hot corona and the dense cool disk from the extended transition layer obtained in our model.

Assuming that both these different models are selfconsistent the question arises which one might be realized in nature. We have seen that the addition of the hot tenuous accreting gas to the already existing layered corona discussed in this paper only constitutes a minor contribution to the dominant coronal evaporation process with its significant radiation 
in the X-ray band. Such coronal evaporation models were successfully used to explain the formation of inner holes in quiescent accretion disks and the soft/hard transition in spectra of soft transients and high-mass X-ray binaries. On the other hand if the tenuous non-radiative accretion would be set up before the standard evaporation could have established itself it might have prevailed until now. This question requires further investigation.

\subsection{A disk in the Galactic Center in the past?}

Assuming the applicability of our modeling we can estimate an upper limit for the mass that might have been left over in a putative accretion disk after a last star forming event, but would now have evaporated by coronal action. No significant X-ray luminosity would then have to be expected today. Such a scenario is not unreasonable since accretion of mass to the central black hole releases angular momentum that is transferred outside in form of matter with Kepler specific angular momentum.

The evaporation rate depends on the value of the viscosity assumed. For a standard value of $\alpha=0.3$ and dominant evaporation, $\eta_{\mathrm{M}}=1$, one has $\dot{M} / \dot{M}_{\text {Edd }} \sim 10^{-2.9}$ corresponding to $\dot{M} \approx 10^{-4} M_{\odot} /$ yr. Observations of stars at the Galactic Center and especially spectroscopy of one such star, S0-2, suggests that these are main sequence O/B stars (Eisenhauer et al. 2003; Ghez et al. 2003). The O/B stars close to the Galactic Center could not have formed longer before their main sequence lifetime, of order of $10^{6.5}-10^{7} \mathrm{yr}$ (Maeder \& Meynet 1989). Thus a disk that remained after the stars had been formed but has evaporated by now should have contained gas not more than 300 to $1000 M_{\odot}$. This value is in the range of mass of the presently observed bright $\mathrm{O} / \mathrm{B}$ stars close to the Galactic Center. Interestingly, but, perhaps by accident, it is also close to the stability limit of a disk against self-gravitation. For an evaporating disk with effective temperature of $50 \mathrm{~K}$ at distance $10^{16.9} \mathrm{~cm}$, the mass of the disk of the stability limit (see e.g., Gammie 2001) is about $10^{3.4} M_{\odot}$.

Thus a disk that had become unstable by self-gravitation and formed the presently observed young massive stars around the Galactic Center until the gravitational instability had ceased could perhaps have now completely disappeared by the process of coronal evaporation. But this remains a rather speculative question until we know more about the stellar population and its origin so close to the Galactic Center.

\section{Conclusions}

We have developed a model for a cool disk around the Galactic Center that has a corona above it, allowing for a coronal accretion of gas captured at the Bondi radius from stellar winds of massive stars. Our models incorporate hydrostatic layering, thermal heat conduction, friction, and radiative energy loss and allow for mass exchange between disk and corona by condensation or evaporation of gas, as well as mass gain or loss by radial flow in the corona (the latter a generalization of the former one-zone model).

We find that the solutions we obtain depend on the value of the viscosity parameter $\alpha$. For standard values, $0.1 \lesssim \alpha \lesssim 0.3$, so much mass evaporates from the disk into the hot corona that the additional mass flow from the outside is a negligible contribution. The evaporation then with time could lead to the complete disappearance of the original disk. Only for values $\alpha \lesssim 0.07$ there might be solutions in which the incoming accreted gas condenses into the disk. However, the vertical structure computations show that in all these solutions the calculated Bremsstrahlung of the corona in the X-ray band by far exceeds the observed luminosity.

From this we conclude that, if our modeling is correct and applicable, at present no cool disk around the Galactic Center exists. We shortly discussed what limit this puts on an inert disk that might have originally remained from a phase of star formation in which the young bright stars presently seen close to the Galactic Center were formed. We also discuss the difference to the alternative non-radiative condensation model of Nayakshin (2004).

Acknowledgements. B. F. Liu would like to thank the Alexander von Humboldt-Foundation for support.

\section{References}

Baganoff, F. K., Maeda, Y., Morris, M., et al. 2003, ApJ, 591, 891

Eisenhauer, F., Schödel, R., \& Genzel, R. 2003, ApJL, submitted [arXiv: astro-ph/0306220]

Esin, A. A., McClintock, J. E., \& Narayan, R. 1997, ApJ, 489, 865

Falcke, H., \& Melia, F. 1997, ApJ, 479, 740

Gammie, C. F. 2001, ApJ, 553, 174

Genzel, R., Pichon, C., Eckart, A., Gerhard, O. E., \& Ott, T. 2000, MNRAS, 317, 348

Genzel, R., Schödel, R., Ott, T., et al. 2003, Nature, 425, 934

Ghez, A. M., Duchene, G., Matthews, K., et al. 2003, ApJ, 586, L127

Ghez, A. M., Morris, M., Becklin, E. E., Tanner, A., \& Kremenek, T. 2000, Nature, 407, 349

Liu, B. F., Mineshige, S., Meyer, F., et al. 2002, ApJ, 575, 117

Liu, F. K., Meyer, F., \& Meyer-Hofmeister, E. 1995, A\&A, 300, 823

Maeder, A., \& Meynet, G. 1989, A\&A, 210, 155

Melia, F., \& Falcke, H. 2001, ARA\&A, 39, 309

Meyer, F., \& Meyer-Hofmeister, E. 1994, A\&A, 288, 175

Meyer, F., \& Meyer-Hofmeister, E. 2002, A\&A, 392, L5

Meyer-Hofmeister, E., \& Meyer, F. 2001, A\&A, 380, 739

Meyer, F., Liu, B. F., \& Meyer-Hofmeister, E. 2000, A\&A, 361, 175

Meyer-Hofmeister, E., \& Meyer, F. 2003, A\&A, 402, 1013

Narayan, R. 2002, in Lighthouses of the Universe, ed. M. Gilfanov, R. Sunyaev, \& E. Churazov (Heidelberg: Springer-Verlag), 405

Narayan, R., Yi, I., \& Mahadevan, R. 1995, Nature, 374, 623

Nayakshin, S. 2003, Astron Nachr/AN 324, No. S1, 3

[arXiv: astro-ph/0302420]

Nayakshin, S. 2004, MNRAS, submitted

Quataert, E. 2003, Astron Nachr/AN 324, No. S1, 3 [arXiv:astro-ph/0304099]

Schödel, R., Ott, T., Genzel, R., et al. 2003, ApJ, 596, 1015

Shakura, N. I., \& Sunyaev, R. A. 1973, A\&A, 24, 337

Smeleva, D. P., \& Syrovatskii, S. I. 1973, Sol. Phys., 33, 341

Spitzer, L. 1962, Physics of Fully Ionized Gases, 2nd edition (New York, London: Interscience Publ.)

Yuan, F., Quataert, E., \& Narayan, R. 2003, ApJ, 598, 301 